\title{
Überlegungen zu einem neuen zweisprachigen phraseologischen Wörterbuch Deutsch-Rumänisch
}

\author{
Doris Sava, Department für anglo-amerikanische und \\ germanistische Studien, Lucian-Blaga-Universität Sibiu, \\ Sibiu, Rumänien (doris.sava@ulbsibiu.ro)
}

\begin{abstract}
Zusammenfassung: Die herkömmliche rumänische zweisprachige Phraseographie mit Deutsch weist erhebliche Mängel auf, die sowohl die konzeptionelle Ausrichtung des Wörterbuchs als auch die Gestaltung der Wörterbuchartikel betreffen. Unter Berücksichtigung der Ergebnisse der bilingualen Speziallexikographie weltweit und der Brauchbarkeit vorhandener Wörterbücher aus heutiger Sicht, ist offensichtlich, dass die Ausarbeitung eines modernen phraseologischen Wörterbuchs, das zuverlässiger und benutzerfreundlicher als bisherige Werke ist, dringend zu fördern ist. Fasst man die Umgestaltung vorhandener Phraseolexika für rumänischsprachige Benutzer des Deutschen ins Auge, so muss das primäre Anliegen der Phraseographen auch darin bestehen, ein Nachschlagewerk auszuarbeiten, das bezüglich der Materialauswahl und der Kodifizierungspraxis höheren Ansprüchen genügen sollte. Der Beitrag zeigt daher Verbesserungsmöglichkeiten der rumänischen bilingualen phraseographischen Beschreibungspraxis mit Deutsch auf, wobei das vorgestellte lexikographische Beschreibungsmuster relevante Informationen zur Rezeption und Produktion der aufgenommenen Phraseologismen bietet.
\end{abstract}

Schlüsselwörter: LEXIKOGRAPHISCHE STANDARDS, PHRASEOLOGISMEN, LEXIKOGRAPHISCHE BESCHREIBUNG, MODELLVORSCHLAG, ZWEISPRACHIGES PHRASEOLOGISCHES WÖRTERBUCH DEUTSCH-RUMÄNISCH

\begin{abstract}
Considerations on a New Bilingual German-Romanian Phraseological Dictionary. The conventional Romanian bilingual phraseography with German contains significant shortcomings regarding both the conceptual orientation of the dictionary and the structure of the dictionary articles. Taking into account the results of specialised bilingual lexicography worldwide and the usefulness of existing dictionaries from a current perspective, it becomes obvious that it is imperative to promote the creation of a modern phraseological dictionary which will be more reliable and user-friendly than the existing works. When the restructuring of the existing phraseological lexica for Romanian-speaking users of the German language is considered, the main concern must be the creation of a reference book which will meet higher requirements regarding the selection of material and the codification practice. Thus, this article points out possible improvements to the Romanian bilingual lexicographical description of phraseological units with German, whereby the presented lexicographical description model offers relevant information for the reception and production of the presented idioms.
\end{abstract}

Lexikos 27 (AFRILEX-reeks/series 27: 2017): 457-477 
Keywords: LEXICOGRAPHICAL STANDARDS, IDIOMS, LEXICOGRAPHICAL DESCRIPTION, PROPOSED MODEL, BILINGUAL GERMAN-ROMANIAN PHRASEOLOGICAL DICTIONARY

\section{Vorbemerkungen}

Die phraseologische und metalexikographische Forschung hat lexikographische Standards für die Erfassung und Darstellung der Phraseologismen in der Wörterbuchpraxis festgelegt, die zu einer Verbesserung der Wörterbücher beigetragen haben. ${ }^{1}$ Die Entwicklung moderner phraseologischer Nachschlagewerke mit Deutsch als Ausgangs- oder Zielsprache ist ein Desiderat. Der Beitrag geht von den Qualitätskriterien für zweisprachige Nachschlagewerke aus, und legt dar, inwieweit eine aus der Sicht der potenziellen Benutzer und der Spezifik phraseologischer Erscheinungen angemessene lexikographische Darstellung gewährleistet werden kann. ${ }^{2}$ Von der Qualität eines Wörterbuchs zeugt weniger die Anzahl der aufgenommenen Lemmata, sondern vielmehr die adressatenspezifische Ausrichtung des Artikelprofils. Die Materialselektion, die Qualität der Wörterbuchangaben sowie Umfang und Konzeption der Umtexte sind weitere Bewertungskriterien, die es zu beachten gilt. Da die rumänische zweisprachige praktische Phraseographie mit Deutsch erhebliche Mängel aufweist, die sowohl die konzeptionelle Ausrichtung des Wörterbuchs als auch die Gestaltung der Wörterbuchartikel betreffen, muss auf die Dringlichkeit eines neuen zweisprachigen phraseologischen Wörterbuchs oder zumindest auf die Notwendigkeit einer Neubearbeitung gegenwärtiger bilingualer Phraseolexika hingewiesen werden. Berücksichtigt man die Ergebnisse der bilingualen praktischen Phraseographie andernorts, so ist zu bedauern, dass hierzulande keine ernsthaften Bemühungen um adäquatere Beschreibungsmöglichkeiten und Kodifizierungsverfahren existieren oder gefördert werden. Die Gründe für dieses Versäumnis sind vielfältig. Sie hängen auch damit zusammen, dass der reichen internationalen (auch germanistischen) wissenschaftlichen Auseinandersetzung mit der phraseologischen Kontrastivik und Phraseographie eine sehr bescheidene Anzahl von Publikationen aus rumänischer Sicht gegenübersteht und dass eine umfassendere Darstellung des rumänischen phraseologischen Systems lange Zeit ausblieb. Die Ermittlung der Vorkommensbereiche und Verwendungsweisen rumänischer Phraseologismen oder Frequenzuntersuchungen zur Produktivität einzelner Komponenten und Strukturmodelle sind Arbeitsfelder, die noch nicht gründlich erforscht wurden. Der Großteil der kontrastiven Untersuchungen aus der Perspektive des anvisierten Sprachenpaares sind dem Bereich der interlingualen Äquivalenzermittlung gewidmet und bezwecken vornehmlich das Vorführen von Besonderheiten, die bei der Kontrastierung bestimmter struktureller Typen hervortreten.

Fasst man die Umgestaltung vorhandener Phraseolexika für rumänischsprachige Benutzer des Deutschen ins Auge, so muss das primäre Anliegen der Phraseographen darin bestehen, ein Nachschlagewerk auszuarbeiten, das bezüg- 
lich der Materialauswahl und Kodifizierungspraxis höheren Ansprüchen genügt. Im Folgenden werden daher phraseologisch relevante Aspekte, die bei der Erstellung oder Revidierung von lexikographischen Werken zu berücksichtigen sind, angesprochen. Dabei sollen an einem Musterartikel Verbesserungsmöglichkeiten für die rumänische bilinguale phraseographische Beschreibungspraxis mit Deutsch exemplarisch aufgezeigt werden, wobei das vorgestellte lexikographische Beschreibungsmuster relevante Informationen zur Rezeption und Produktion der aufgenommenen Phraseologismen bietet.

Mit der Festlegung des Benutzerkreises und seiner Informationsbedürfnisse ist die Gestaltung der Wörterbuchstruktur und der Informationstypen verbunden, die in der Mikrostruktur vorkommen. Ein zweisprachiges phraseologisches Wörterbuch muss dem Benutzer u.a. folgende Informationen liefern: (1) In welchen Situationen wird der Phraseologismus gebraucht? (2) Können alle Äquivalente in der gleichen Situation gebraucht werden wie der ausgangssprachliche Phraseologismus? (3) Welche Besonderheiten zeigen die Phraseologismen in der Ausgangssprache?

Bei der Erwartungshaltung des Wörterbuchbenutzers muss in Erwägung gezogen werden, dass der Wörterbuchbenutzer zum Wörterbuch greift, um einen Ausdruck in seiner Form bzw. in seinen Formen wie auch in seiner Bedeutung und Verwendungsweise im aktuellen Sprachgebrauch zu überprüfen. So ein Wörterbuch könnte ein passives Verständnis sichern, im Optimalfall auch eine aktive Verwendung bzw. auch zur Textproduktion anleiten und nicht nur rezeptiv ausgerichtet sein. Die lexikographischen Informationen müssen hinsichtlich der (tatsächlichen und potenziellen) Benutzungssituationen formuliert werden. Bei der Konzeption eines zweisprachigen phraseologischen Wörterbuchs mit Deutsch und Rumänisch wird von der Prämisse ausgegangen, dass das Nachschlagewerk sowohl für die Textrezeption als auch indirekt für die Textproduktion von rumänischen Deutschlernern sowie sekundär für die Textrezeption von deutschsprachigen Rumänischlernern genutzt werden kann.

Ein deutsch-rumänisches Wörterbuch kann von zwei verschiedenen Benutzergruppen zum Nachschlagen herangezogen werden. Daraus ergeben sich zwei unterschiedliche Benutzungssituationen, je nachdem, ob die Muttersprache des Benutzers die Ausgangssprache oder die Zielsprache darstellt, in der Informationen zu bestimmten Phraseologismen gesucht werden. Ein rumänischsprachiger Benutzer wird einen Phraseologismus in einem phraseologischen Wörterbuch Deutsch-Rumänisch nachschlagen wollen, (1) weil er die Bedeutung aus einem gegebenen gesprochenen/geschriebenen Text selbstständig nicht erschließen kann, auch wenn er die Bedeutung einzelner Konstituenten kennt. Er sucht eine Information über einen Phraseologismen in der Ausgangssprache Deutsch; (2) weil er bei einer Übersetzung in die Muttersprache Rumänisch ein Äquivalent sucht und das Wörterbuch eine zielsprachliche Entsprechung liefern sollte; (3) weil der rumänischsprachige Benutzer zwar die Bedeutung eines Phraseologismus kennt, jedoch unsicher ist, was seine ange- 
messene kontextuelle Einbettung oder die vollständige/richtige lexikalische Form anbelangt und deshalb danach nachschlagen möchte. Ein Benutzer mit Deutsch als Muttersprache hingegen wird aus anderen Gründen ein phraseologisches Wörterbuch Deutsch-Rumänisch konsultieren wollen. Er verfügt bereits über eine muttersprachliche idiomatische Kompetenz in der Ausgangssprache und sucht gezielt nach Informationen über mögliche zielsprachliche Entsprechungen zu bestimmten Phraseologismen. Dabei kann mit folgenden Benutzungssituationen gerechnet werden: (1) der deutschsprachige Benutzer sucht ein Äquivalent für einen Phraseologismus, der in einem gesprochenen/ geschriebenen Text in der Zielsprache Rumänisch vorkommt und benötigt eine Äquivalentangabe für die Hinübersetzung; (2) der Benutzer möchte einen fremdsprachlichen Text produzieren, weiß jedoch nicht, wie er den muttersprachlichen Inhalt phraseologisch in der Zielsprache ausdrücken kann.

Nach der in der zweisprachigen Lexikographie üblichen Wörterbuchtypologie soll das vorgeschlagene Wörterbuchprojekt als bidirektionales Konsultationswörterbuch (Nachschlage- bzw. Bestätigungsfunktion), primär rezeptiv aufgefasst werden, das jedoch auch weiteren Anforderungen nachkommen kann, wie z.B. der Lernfunktion. Es sollte Situationen der Textrezeption und der Herübersetzung abdecken, kann jedoch auch als aktives Wörterbuch aufgefasst werden.

2. Das Gebot der Kürze und die Verlockungen der Ausführlichkeit. Phraseologismen in der lexikographischen Praxis

\subsection{Defizite der rumänischen bilingualen Phraseographie mit Deutsch im Überblick}

In der lexikographischen Forschung wurde die Frage nach den Bedürfnissen des Benutzers zweisprachiger Wörterbücher intensiv diskutiert, vor allem die nach der angemessenen Auswahl und Darstellung der für die jeweilige Benutzergruppe relevanten Informationen. Die kritische Durchsicht der Wörterbucheintragungen hat Überlegungen zu einer sorgfältigeren Beschreibung der Phraseologismen veranlasst. Die Bemühungen der Lexikographen sind auf eine möglichst umfassende Beschreibung der semantischen Komplexität, formalen Vielgestaltigkeit und pragmatischen Vieldeutigkeit ausgerichtet. Dabei treten deutlich die Schwierigkeiten hervor, die mit diesem Unterfangen verbunden sind, wie auch offensichtlich wird, dass ein theoretisch-methodisch fundiertes und auch praktisch umsetzbares lexikographisches Konzept notwendig ist, das auch die Forschungsergebnisse der theoretischen und praktischen, allgemeinen und kontrastiven Phraseologie zu berücksichtigen hat. Die dem Lexikographen von der theoretischen bzw. praktischen Phraseographie zur Verfügung gestellten Lösungen wurden unterschiedlich gewertet: als aufwendig, subjektiv und nicht konsequent durchführbar, wenn auch benutzerfreundlich. ${ }^{3}$ In einigen Beiträgen (z.B. 2010) bin ich auf die rumänische zwei- 
sprachige praktische Phraseographie mit Deutsch als Ziel- und Ausgangssprache $^{4}$ eingegangen, wobei ich die in Rumänien ausgearbeiteten Wörterbücher aus der Perspektive des Nichtmuttersprachlers und seiner Benutzerbedürfnisse bewertet habe. Die durchgesehenen Wörterbücher verzeichnen Phraseologismen alphabetisch, wobei jedem Lemma Äquivalentangaben mit usueller Variantenkennzeichnung zugeordnet werden: $a$ cădea/a da/a sări din lac in puţ - aus dem/vom Regen in die Traufe kommen (Mantsch/Anuței/Kelp 1979: 289) vs. vom/aus dem Regen in die Traufe kommen - a da din lac în puţ (Roman 1993: 203 und 262). Insgesamt ist die lexikographische Versorgung in diesen Lexika als mittelmäßig bis schlecht zu beurteilen. Diese Einschätzung sei im Folgenden näher begründet.

Bei der Musterung beider Wörterbücher sind zahlreiche Ungenauigkeiten und Verstöße gegen die lexikographische Akribie auszumachen. Roman (1993) geht inkonsequent vor, wenn er im Vorspann Beispiele vorstellt (z.B. von dem Regen in die Traufe kommen; S. 5), die im Wörterbuchkörper mit Variantenkennzeichnung kodifiziert werden: vom/aus dem Regen in die Traufe kommen (1993: 203 und 262). Bereits im Vorspann begegnen bei Roman einige (Druck-)Fehler und zwar genau in dem Textabschnitt, der den Benutzerhinweisen gewidmet ist und wo der Autor die Prinzipien der lexikographischen Erfassung erläutert. Zur Veranschaulichung wählt Roman einen Phraseologismus, der zwei nominale Elemente aufweist, um darzulegen, unter welcher wendungsinternen Komponente der Phraseologismus gebucht wird. Der deutsche Phraseologismus vom/aus dem Regen in die Traufe kommen erscheint an zwei Stellen im Wörterbuch, wird aber nur unter dem ersten Substantiv mit seiner rumänischen Entsprechung angeführt und unter der weiteren Komponente Traufe allerdings in der Form Von dem Regen in die Traufe Kommen (sic!) - mit dem Verweis „V. Regen“ [v. = rum. vezi; dt. siehe] versehen. Im Wörterbuch allerdings erscheint der Phraseologismus ohne Druckfehler. Gleichfalls inkonsequent gehen auch Mantsch/Anuţei/Kelp (1979) vor. Vgl. hierzu die deutschen Entsprechungen zu a cădea din lac în puţ und a cădea/a da/a sări din lac în puţ aus dem/vom Regen in die Traufe kommen (Mantsch/Anuţei/Kelp 1979: 104 und 289); colac peste pupăză - aus dem Regen in die Traufe kommen (Mantsch/Anuței/Kelp 1979: 137); a căuta pe dracul şi a găsi pe tată-său vom/aus dem Regen in die Traufe kommen (Mantsch/Anuţei/Kelp 1979: 173) und auch folgende Äquivalenzangaben: „für zwei arbeiten, wie ein Pferd arbeiten" (S. 311), "schwer arbeiten, wie ein Pferd/Galeerensklave arbeiten, Blut und Wasser schwitzen, etwas ist ein saurer Verdienst" (S. 233), "schuften, sich abrackern, sich schinden, sich (ab)mühen, sich placken, wie ein Pferd/Ochse arbeiten, Pferdearbeit verrichten" (S. 243) und "schwer arbeiten, sich placken, sich schinden, in den Sielen stecken, sich ins Joch spannen" (S. 289). Gravierend ist insbesondere die Tatsache, dass manche Phraseologismen in verschiedenen Nennformen lexikographisch erfasst worden sind. Nichtmuttersprachliche Wörterbuchbenutzer benötigen ein Maximum an Informationen, wenn das zweisprachige Wörterbuch den Anspruch erhebt, ein Hilfsmittel für die Text- 
produktion zu sein. Damit die Nennform eine sichere Textproduktion gewährleisten kann, muss durch die Wahl der Nennform klare Aussagen über mögliche Restriktionen in der Verwendung der Phraseologismen gemacht werden. Daher sollte eine benutzergerechte Nennformgestaltung dem Benutzer die wichtigsten Informationen zur Einbettung des Phraseologismus im Satz liefern und syntaktisch-semantische Angaben zu den Kontextpartnern (zu den obligatorischen und fakultativen Valenzen der Phraseologismen) als Informationstyp explizit und vollständig darbieten. Aus der Analyse der Nennformen geht hervor, dass der Wörterbuchbenutzer unterschiedliche Informationen darüber findet, welche Komponenten einen Phraseologismus ausmachen. So verzeichnen Mantsch/Anuţei/Kelp (1979: 229) als deutsche Entsprechung für acesta este adevărul gol-goluţ "das ist die nackte/reine Wahrheit”. 22 Zeilen darunter erscheint unter a spune adevărul gol-goluţ das Äquivalent "die nackte/reine/ ungeschminkte Wahrheit sagen". Durch die Vernachlässigung der Information zur Valenzfähigkeit des Phraseologismus und die Reduzierung seiner Nennform kann der Wörterbuchbenutzer nicht immer einschätzen, ob als Handlungsträger eine Person oder auch eine Institution, Einrichtung usw. genannt werden kann. Die Nennformgestaltung sollte Restriktionen kenntlich machen und die korrekte Nennform sollte so formuliert werden, dass Angaben zur externen syntaktisch-semantischen Valenz auch berücksichtigt werden. Für die Klärung der Frage nach den fakultativen und obligatorischen Komponenten des Phraseologismus, die in die Nennform aufgenommen werden sollen, und für eine vollständige und korrekte Kodifizierung sind Recherchen der modernen elektronischen Textkorpora wie auch das Sammeln von Belegen aus modernen Quellen (z.B. aus der aktuellen Presselandschaft) hilfreich. Roman (1993) lässt die externe Valenz oft außer Acht und konkretisiert die Subjektergänzung in der Nennform nicht. Irrtümlicherweise verzeichnet Roman neben den in der lexikographischen Praxis üblichen Abkürzungen etw. (= etwas), jd. (= jemand) und js. (= jemandes) in dem Abkürzungsverzeichnis das Kürzel jm. mit der Erklärung jemanden (!). Die Abkürzung jn. (= jemanden) wurde nicht aufgenommen. Konsequent begegnet man im Wörterbuch dem Element jemandem mal in der Abkürzung jm. oder jn., sodass auch falsche Nennformangaben vorkommen: jm. (blauen/eitlen) Dunst vormachen; jm. nicht um die Ecke trauen (S. 60f.); jm. das Fell über die Ohren ziehen (S. 77); jm. mit (eisiger) Kälte empfangen (S. 134). $\mathrm{Zu}$ bemängeln ist auch, dass zwei oder mehrere Phraseologismen in einer einzigen Nennform zusammengefasst werden. Bei Mehrfachlemmatisierungen stimmen Nennformangaben, Markierungen und Äquivalenzangaben oft nicht überein. Als zielsprachliche Äquivalente fungieren Phraseologismen, freie Wortverbindungen, Umschreibungen oder Einwortlexeme, Sprichwörter oder Goethe-Zitate (!). Roman (1993) ordnet in fünf Fällen Sprichwörtern phraseologische Wortverbindungen (S. 216, 240, 267, 292) zu, wobei bekannte Sprichwörter (z.B. Der Ton macht die Musik; S. 260; Übung macht den Meister; S. 267) ohne den Hinweis proverb (dt. Sprichwort) erscheinen. Bei Mantsch/Anuţei/Kelp (1979) werden 194 Sprichwörter ohne diesen Hinweis (vgl. S. 73, 82f., 105, 228, 
326, 365 oder 613; Aus den Augen, aus dem Sinn; Die Katze lässt das Mausen nicht; Es ist nicht alles Gold, was glänzt; Aller Anfang ist schwer; Ende gut, alles gut; Eile mit Weile; Der Apfel fällt nicht weit vom Stamm; Kleider machen Leute; Wer A sagt, muss auch $B$ sagen) aufgenommen. Hier werden deutsche Sprichwörter in verschiedenen Varianten angeführt (vgl. z.B. S. 149, 218 oder 557) und auch bei Roman werden 39 deutsche Sprichwörter in variierter Form dargeboten: z.B. Über Nacht kommt Rat; guter Rat kommt über Nacht (S. 179) oder Auf Regen folgt Sonnenschein; nach Regen kommt Sonnenschein (S. 203).

Die Sichtung dieser Wörterbücher zeigt, dass sich pragmatische Informationen eher auf stilistische Markierungen beschränken. Insgesamt kommen geschlechts- oder altersspezifische Markierungen äußerst selten vor. Oft in Form von Abkürzungen geboten, verzeichnen die Wörterbücher in den Vorworten nicht immer, was unter den betreffenden Stilangaben zu verstehen ist. In beiden Wörterbüchern können weitere Versäumnisse registriert werden, die den Wörterbuchbenutzer stören werden. Sowohl bei Mantsch/Anuţei/Kelp (1979) als auch bei Roman (1993) erscheinen Abkürzungen, die im Verzeichnis nicht erfasst wurden. Bei Mantsch/Anuţei/Kelp kommen im Wörterbuch Abkürzungen wie prov. (rum. proverb; dt. Sprichwort) oder înv. (rum. invechit; dt. veraltet) vor, die im Verzeichnis nicht erscheinen. Verwunderlich ist, dass die Autoren 37 Phraseologismen mit dem Hinweis fig. (rum. figurat; dt. bildhaft; übertragen, umgedeutet) versehen, diese Abkürzung jedoch in die Liste der Abkürzungen nicht aufnehmen. Bei Roman erscheinen sieben Abkürzungen für Markierungsprädikate oder Vorkommensbereiche, die im Vorspann nicht erfasst wurden (z.B. die Markierung arg. für rum. argou, argotic; dt. Gaunersprache). Ein Inhaltsverzeichnis als äußere Zugriffsstruktur zum gesamten Textverbund oder ein Index der Schlüsselwörter, der das Auffinden kodifizierter phraseologischer Einheiten ermöglichen soll, vermisst man in beiden phraseologischen Wörterbüchern.

Das lexikographische Bild rumänischer Phraseolexika verweist auf eine dem erfassten Sprachbestand nicht angemessene lexikographische Bearbeitung. Fasst man bestimmte lexikographische Standards ins Auge, so kann auch von einer sorgfältigen Ausführung der Umtexte bei diesem speziellen Wörterbuchtyp nicht gesprochen werden. Beide Wörterbücher liegen unter dem Gesichtspunkt der Gestaltung der Einleitung weit unter dem Stand eines benutzerfreundlichen Nachschlagewerks. Sie enthalten zwar im rumänischsprachigen Vorspann ein kurzes Vorwort, das neben den Bemerkungen zum Adressatenkreis und zu usuellen Benutzungssituationen auch Auskunft über den erfassten phraseologischen Wortschatzausschnitt liefert, aber insgesamt beschränkt sich der Vorspann auf eine kurze Benutzeranleitung und auf die Erläuterung der Abkürzungen im Artikeltext. Die Vorspannstrukturen beider Wörterbücher sind bezüglich der Anzahl und der Funktion ihrer Teile ähnlich. Außer einem Vorwort und einem Abkürzungsverzeichnis weist nur Mantsch/Anuţei/Kelp (1979) in einem gesonderten Abschnitt explizite auf Benutzungshinweise hin, wie auch nur bei diesem Nachschlagewerk ein Verzeichnis der herangezo- 
genen Lexika und Quellen vorhanden ist. Mantsch/Anuței/Kelp legen einen ausführlicheren Vorspann als Roman vor, der auch klassifikatorische Schwerpunkte berücksichtigt und mit Beispielangaben untermauert. Positiv zu bewerten ist die dargebotene Bibliographie, die man bei Roman (1993) vermisst. Die Auflistung der Fachquellen und der zitierten Werke, der Verweis auf Parallelunternehmen oder die weiterführende Bibliographie sollen nicht als Sammelsurium zufälliger Titel fungieren, sondern auf die wichtigsten Titel begrenzt sein.

Die gesichteten Lexika bringen knappe Informationen zur Kodifizierungspraxis und wenige Hinweise zur lexikographischen Bearbeitung von speziellen Phraseologismustypen und den daraus sich ergebenden Konsequenzen. Da Phraseologismen mehrgliedrig sind, besteht eine grundsätzliche Schwierigkeit darin, zu entscheiden, unter welchem Stichwort ein Phraseologismus lexikographisch erfasst werden soll. Eine benutzerfreundliche Makrostruktur geht von formalen Kriterien aus, die bei der Kodifizierung von Phraseologismen auch strikt eingehalten werden sollten. In der Einleitung sollte ein präzises Anordnungsschema ausführlich vorgeführt und erklärt werden, sodass der Benutzer daraus erschließen kann, wie der jeweilige Phraseologismus im Wörterbuch zu finden ist. Die aufgezeigten Schwächen beeinflussen die Wörterbuchbenutzung in rezeptiven wie in produktiven Nachschlagesituationen. Sie sind auch durch die Spezifik phraseologischer Erscheinungen (u.a. semantischpragmatische Komplexität und polylexikalischer Charakter) erklärbar.

In den rumänischen Phraseolexika sind die speziellen Prinzipien der lexikographischen Erfassung des phraseologischen Inventars und damit auch die praktischen Bedürfnisse der Wörterbuchbenutzer ungenügend berücksichtigt. Die Konzeption der untersuchten Wörterbücher ist im Hinblick auf die lexikographische Makro- und Mikrostruktur zu wenig auf benutzerorientierte bzw. benutzungsbezogene Zielsetzungen ausgerichtet. Das bedeutet, dass die verschiedenen lexikographischen Angaben und die Wörterbuchartikel für eine Neuauflage anders strukturiert und die lexikographischen Informationen hinsichtlich der tatsächlichen und potenziellen Benutzungssituationen neu konzipiert und formuliert werden müssen. Es müssen nicht nur die Umtexte ausgeweitet und inhaltlich dem aktuellen Stand der Forschung angepasst werden, sondern auch die Auswahl, die Anordnung und lexikographische Bearbeitung der Phraseologismen in den herkömmlichen rumänischen phraseologischen Wörterbüchern müsste hinterfragt werden. ${ }^{5}$ Einige der hier aufgezeigten Versäumnisse hinsichtlich der lexikographischen Konzeption und Ausgestaltung der Wörterbuchartikel hängen weniger mit den lexikographischen Entscheidungen der Wörterbuchautoren zusammen, sondern sind in einem verlegerischen Kalkül begründet.

Für Neuauflagen oder für ausstehende Nachschlagewerke zum Sprachenpaar Deutsch und Rumänisch müssen die hier angesprochenen Mängel behoben werden, wobei eine stärkere Berücksichtigung der Benutzerbelange und der in der Forschung postulierten Anforderungen an die bilinguale phraseographische Praxis mit Deutsch zu erwarten ist. Für die praktische bilinguale 
Phraseographie der Sprachen Deutsch und Rumänisch ist daher die Ausarbeitung eines modernen phraseologischen Wörterbuchs, das zuverlässiger und benutzerfreundlicher als bisherige Werke ist, dringend zu fördern.

\subsection{Zur Konzeption eines neuen zweisprachigen phraseologischen Wör- terbuchs Deutsch-Rumänisch}

Das vorgeformte Sprachgut umfasst unterschiedlich gestaltete Einheiten und ist folglich sehr heterogen. Es nimmt eine Sonderstellung im Sprachsystem und -gebrauch ein, insofern es sich durch bestimmte inhaltliche, formale und pragmatische Besonderheiten von den übrigen Inventareinheiten der Sprache abhebt. Ausgewählte Typen von Phraseologismen sind für bestimmte Kommunikationsbereiche und Textsorten konstitutiv, was die Berücksichtigung ihres semantisch-pragmatischen Anwendungspotenzials bedingt und dessen aktive Aneignung und lexikographische Erfassung erheblich erschwert. Das rege Interesse an Fragestellungen der kontrastiven Phraseologie - nicht nur im binnendeutschen Sprach- und Kulturraum, sondern auch andernorts - ist auch in der Auseinandersetzung mit Problemstellungen zur Phraseographie verschiedener Sprachen zu suchen. Als Resultat zahlreicher theoretischer wie auch praktischer Auseinandersetzungen mit Fragestellungen aus dem Bereich Phraseologismen und Lexikographie ist u.a. die Notwendigkeit einer Neuorientierung gefordert worden, wobei nach Burger (1992) zweierlei abgewogen werden muss: (a) die gängige Wörterbuchpraxis und (b) die Erkenntnisse der Phraseologie-Forschung, da von hier aus konkrete Forderungen an die lexikographische Praxis gestellt wurden, die zu einer verbesserten Qualität der Wörterbücher beitragen sollen. Schemann (1989: 1026) erachtet es als die „vielleicht schwierigste Aufgabe" des Verfassers eines phraseologischen Wörterbuchs, dem Benutzer all jene grammatischen Informationen zu liefern, die er benötigt, um einen phraseologischen Ausdruck in jedem möglichen Kontext korrekt zu gebrauchen.

Die moderne Metalexikographie versucht, durch die Bereitstellung eines geeigneten Methodenapparats und die Ausarbeitung neuer Kodifizierungsverfahren den Benutzerbelangen und der adressatengerechten Ausrichtung der Wörterbücher zu dienen, um künftige Wörterbücher oder neue Wörterbuchauflagen zu verbessern. Dabei ist darauf verwiesen worden, dass eine verbesserte Adressatenorientiertheit nicht nur durch einen sorgfältigen mikrostrukturellen Aufbau gesichert werden kann, sondern auch durch eine einheitliche lexikographische Bearbeitung.

Voraussetzung für die Zusammenstellung eines benutzerfreundlichen Nachschlagewerks sind theoretische und praktische Überlegungen zum Benutzerprofil, zu Auswahl- und Darstellungskriterien des kodifizierten Sprachmaterials, eine sorgfältige Erfassung der Gebrauchsbeschränkungen im aktuellen Sprachgebrauch wie auch eine verbesserte Äquivalentdarstellung. Für Neuauflagen vorhandener Wörterbücher zum Sprachenpaar Deutsch und 
Rumänisch oder für die Konzeption geplanter ist die Ausarbeitung einer adäquaten Beschreibungsweise zwingend und eine eingehendere empirische Fundierung ${ }^{6}$ notwendig, um eine angemessene lexikographische Darstellung phraseologischer Einheiten zu gewährleisten. Aus der Perspektive dieses Sprachenpaares gestaltet sich dieses Vorhaben als schwierig, insofern eine Metasprache erst ausgearbeitet werden muss, da kein differenziertes und auf lexikographische Zwecke ausgerichtetes metalexikographisches Beschreibungsvokabular im Rumänischen bereitsteht. Eine Neubearbeitung ist auch dadurch erschwert, dass die Eintragungen relevante Informationen zur Rezeption und/ oder Produktion der kodifizierten Phraseologismen enthalten müssen, die eine situations- und textsortenangemessene wie partnergerechte Verwendung des kodifizierten Sprachmaterials gewährleisten sollen, um eine aktive Aneignung des phraseologischen Inventars zu unterstützen. ${ }^{7}$

Für den Benutzer eines bilingualen phraseologischen Wörterbuchs sind bestimmte Informationen relevant: (a) die Lemmazuordnung; (b) die Nennformgestaltung; (c) die Vorkommensbedingungen; (d) die Äquivalenzdarstellung. Im Hinblick auf eine verbesserte Beschreibungspraxis des anvisierten Sprachenpaares sollte danach getrachtet werden, nicht nur zielsprachliche Äquivalente zu erfassen, sondern auch Charakteristika der kodifizierten Einheiten. Daher müssten Erweiterungen der Einträge um bestimmte Artikelpositionen eingeplant werden. Von grundlegender Bedeutung sind hierbei die Angaben zur sprachlichen Umgebung und die Kennzeichnung der stilistischpragmatischen Besonderheiten. Über das Verstehen einer festen Wortverbindung hinaus muss die lexikographische Beschreibung auch den korrekten Gebrauch der kodifizierten Phraseologismen bei der Textproduktion sicherstellen. Das Wörterbuchkonzept, das hier in Grundzügen vorgestellt werden soll, fasst idiomatisierte Phraseologismen ins Auge und richtet sich an einen breiten Adressatenkreis (z.B. Studierende, Lehrkräfte, Lehrbuchautoren, Übersetzer). Dabei wird von der Prämisse ausgegangen, dass das Nachschlagewerk sowohl für die Textrezeption als auch indirekt für die Textproduktion von rumänischen Deutschlernern sowie sekundär für die Textrezeption von deutschsprachigen Rumänischlernern genutzt werden kann. Bei der Konzeption eines Beschreibungsmodells mit Deutsch als Ausgangssprache für rumänische Benutzer wird eine Auffassung von Phraseologie im engeren Sinne vertreten, um eine möglichst einheitliche und umfassende Beschreibung der kodifizierten deutschen Phraseologismen mit ihren rumänischen Entsprechungen $\mathrm{zu}$ gewährleisten. Sie steht auch in Einklang mit den Empfehlungen der bilingualen Metalexikographie. ${ }^{8}$

Für die Ausarbeitung eines modernen und benutzerfreundlichen Beschreibungsmodells wurden die lexikographischen Standards, die die metalexikographische Forschung hinsichtlich Adressatengruppen und deren Nutzungsinteressen formuliert hat, berücksichtigt wie auch einige Verbesserungsvorschläge, die eine Erweiterung der Einträge um bestimmte Artikelpositionen gefordert haben. Für diesen speziellen Wörterbuchtyp hat Cheon (1998) Über- 
legungen zum Ausbau der Mikrostruktur formuliert, während Mudersbach (1998) eine Beschreibung aufgrund eines universalen pragmatischen Modells vorschlägt, das das enthalten sollte "was ein Nichtmuttersprachler wissen muss, um ein Phrasem in einer Situation richtig gebrauchen zu können." Hier muss betont werden, dass der Einbezug von Phraseologen bei der Revidierung von Wörterbüchern notwendig ist, wie auch die Berücksichtigung der Ergebnisse der theoretischen und angewandten allgemeinen und kontrastiven Phraseologie, um eine optimale Lemmatisierung und Anordnung des kodifizierten phraseologischen Bestandes gewährleisten zu können. ${ }^{9}$

Die vorhandenen Nachschlagewerke zum Sprachenpaar Deutsch und Rumänisch, die in Rumänien erarbeitet worden sind, sind veraltet. Bei dem damaligen Stand der Phraseologie/Lexikographie - insbesondere der zweisprachigen - ist die mangelhafte lexikographische Bearbeitung phraseologischer Einheiten nachvollziehbar. Bei der Ausarbeitung eines Verbesserungsvorschlags ${ }^{10}$ zur phraseographischen Praxis des Sprachenpaares Deutsch und Rumänisch waren folgende Überlegungen ausschlaggebend: (1) das Erarbeiten eines einheitlichen Beschreibungsmodus; (2) die Präsentierung und Gestaltung der Information, die ein semantisches-pragmatisches Verständnis gewährleisten sollte, im Hinblick auf die Erleichterung der Textproduktion und -rezeption; (3) die Ausarbeitung einer Metasprache, welche die Kriterien der Darstellung des phraseologischen Materials verdeutlichen und die Besonderheiten des aufgenommenen Inventars angemessen erfassen sollte. Bei der Erarbeitung eines bilingualen phraseologischen Wörterbuchs muss in Erwägung gezogen werden, dass für den Benutzer bestimmte Informationen relevant sind. Deshalb sollte der Wörterbuchvorspann - über das Verzeichnis der verwendeten Zeichen, Abkürzungen usw. hinaus - Angaben zum kodifizierten phraseologischen Inventar mit Hinweisen zum terminologischen und definitorischen Ansatz, Informationen zur Selektion und Präsentation phraseologischer Einheiten, zur Kennzeichnung der Gebrauchsweisen und Angaben zur Äquivalenzerfassung und -gestaltung enthalten. Um die aktive Aneignung von Phraseologismen zu unterstützen, wird der alphabetische Wörterbuchteil ergänzt durch einen Indexteil, der einen raschen Zugriff auf den gesuchten Phraseologismus ermöglicht und dem Wörterbuchbenutzer verdeutlicht, wie er das Nachschlagewerk konsultieren kann und soll. Dieser Indexteil umfasst folgende Teile: (a) ein alphabetisches Register, das alle im Wörterbuch verzeichneten und beschriebenen Phraseologismen in alphabetischer Reihenfolge aufführt und die entsprechende Seitenanzahl im Wörterbuch angibt. Das alphabetische Register ist praktisch für jede Benutzungssituation erforderlich und dient speziell den Zwecken des Nachschlagens bei der Textrezeption. Dieser Wörterbuchteil ermöglicht einen schnellen Zugriff auf unbekannte Phraseologismen. Er bietet eine Lernmöglichkeit im Sinne der Kontrolle und Wiederholung; (b) ein Register zielsprachlicher Äquivalente, falls der Benutzer eine dem rumänischen Phraseologismus entsprechende deutsche Wendung sucht; (3) ein Register alphabetischer Leitbegriffe ermöglicht über einen onomasiologischen Ansatz 
den Zugang zu den rumänischen Phraseologismen. Dieser Wörterbuchteil kann mit bestimmten Einschränkungen die Funktion eines aktiven Wörterbuchs übernehmen. Konzeptionell würde ein moderneres phraseologisches Wörterbuch Deutsch-Rumänisch aus einer alphabetischen Darstellung des erfassten phraseologischen Wortschatzes und einem Indexteil bestehen.

Der Verbesserungsvorschlag wird daher folgende phraseographisch vorbelastete Problembereiche anvisieren: die Umtextgestaltung, die Nennformgestaltung, die Darbietung der Restriktionen und die Markierungspraxis. Die anvisierten Neuerungen der phraseographischen Praxis betreffen folglich: (1) die Orientierung im Wörterbuch; (2) die lexikographische Beschreibungspraxis, d.h. die lexikographische Erfassung und Darstellung phraseologischer Spezifik (Nennformgestaltung; Präsentation phraseologischer Variation; Berücksichtigung der externen Valenz; Erfassung spezifischer Gebrauchsmuster samt Darbietung der formal-semantischen und pragmatischen Restriktionen; verbesserte Markierungspraxis); (3) die primäre Berücksichtigung der Belange von Deutschlernenden.

Bei der Nennformgestaltung müssen dem Wörterbuchbenutzer Angaben geliefert werden, um die entsprechende phraseologische Einheit auch aktiv verwenden zu können, das bedeutet, dass morphosyntaktische Restriktionen kenntlich gemacht werden müssen. ${ }^{11}$ Mit der Angabe der Nennform werden dem Benutzer die wichtigsten Informationen zur Einbettung des Phraseologismus im Satz geliefert. Um auch der Lernfunktion gerecht zu werden, muss die Nennform gewisse Voraussetzungen erfüllen. Dies bedingt, dass die Nennform Informationen über die Kontextpartner, d.h. über die obligatorischen und fakultativen Valenzen der Phraseologismen liefern soll. Die Nennform sollte in einer neutralen, nicht aktualisierten Form aufgeführt werden, wenn keine morphosyntaktischen Restriktionen vorliegen. Um vollständig zu sein, muss die Nennform mit den entsprechenden externen Valenzen ausformuliert werden. Das Indefinitpronomen etwas kann in der Nennform als metasprachliche Angabe fungieren, um die Valenz anzuzeigen, wobei etwas immer mit einem variablen Element in der Kommunikation zu ersetzen wären, zum anderen geht es um eine invariable Komponente der phraseologischen Einheit. Die Abkürzung des Indefinitpronomens etw. wird daher zur Markierung der unbelebten externen Valenz eingesetzt, sodass der Unterschied zwischen wendungsinternem Indefinitpronomen (z.B. nach etwas aussehen) und wendungsexterner Valenz (z.B. etw. mit der Muttermilch einsaugen) eindeutig ist. Elemente des Typs jmdm., jmdn., jmds., jmd., etw. gehören zu den obligatorischen Komponenten des Phraseologismus. Damit werden sowohl semantische Kriterien (u.a. Personen- und Sachbezug) als auch syntaktische Kriterien bei der Einbettung fester Wortverbindungen in den Ko(n)text berücksichtigt. Für Phraseologismen, die ein obligatorisches Subjekt als Komponente enthalten, hält es die lexikographische Forschung aus der Sicht des Fremdsprachenlerners angemessen, bei der lexikographischen Darstellung die Präsensform anzuführen. Dadurch erscheint die für den Lerner wichtige Ergänzung in Subjektposition in der 
Nennform. Für die Frage nach den fakultativen und obligatorischen Komponenten des Phraseologismus, die in die Nennform aufgenommen werden sollen, können für eine vollständige und korrekte Kodifikation Recherchen der modernen elektronischen Textkorpora wie auch das Sammeln von Belegen aus modernen Quellen (z.B. aus der aktuellen Presselandschaft) hilfreich sein.

Bei der lexikographischen Darstellung der Phraseologismen empfiehlt sich, den sprachlichen und den situativen Kontext (pragmatische Angaben/ gebrauchssemantische Beschreibung) zu berücksichtigen. Des Weiteren ist davon auszugehen, dass man den semantischen Inhalt eines Phraseologismus nicht ohne stilistische und pragmatische Informationen erfassen kann. Daher sind Gebrauchsmuster in einem phraseologischen Wörterbuch notwendig. Burger (1992: 49) schlägt standardisierte Formulierungen für die Erfassung pragmatischer Aspekte in der lexikographischen Praxis vor, die im Wörterbuchvorwort zu erläutern sind.

Die metalexikographische Fachliteratur hat eine Reihe von Überlegungen und Empfehlungen zu einer konsistenteren Markierungspraxis angestellt bzw. ein offenes Beschreibungsinventar vorgeschlagen.12 Die Fachliteratur hat empfohlen, die Bedeutungserklärung durch pragmatische Hinweise zu ergänzen, um die für den Nichtmuttersprachler wichtigen Aspekte des Phraseologismusgebrauchs (Situationsspezifik, Stilmarker, Sprecherintention, emotionaler Gehalt, Geltungsbereich) erfassen zu können. Vgl. hierzu folgende Angaben: Charakterisierung der Gebrauchssituation ( \pm mündlich/schriftlich); Sprechereinstellung (z.B. euphemistisch/verhüllend, scherzhaft/humorvoll, pejorativ/ironisch/ spöttisch/geringschätzig, vorwurfsvoll/verächtlich, grotesk/derb, abwertend, feierlich, beschönigend); Emotionalitätsgrad (z.B. \pm übertrieben/ emotional); Stilschichtmarkierung (z.B. gehoben, umgangssprachlich, salopp); Charakterisierung der Sprecher-Hörer-Konstellation ( \pm Vertrautheit, \pm formell, \pm hierarchisch, \pm symmetrisch).

Pragmatische Erkenntnisse haben zwar einen "breiten Eingang“ in die Theorie der Phraseographie gefunden, ,es mangelt jedoch an der konsequenten praktischen Umsetzung der gewonnenen Postulate" (Filatkina 2007: 152) in der allgemeinen und phraseologischen Wörterbuchpraxis. Angesichts der Tatsache, dass in den herkömmlichen Wörterbüchern die Semantik der Phraseologismen oft - und fast ausschließlich - durch deren denotative Bedeutung erfasst wird, hat sich in der metalexikographischen Diskussion verstärkt die Forderung nach einer sprachhandlungstheoretisch orientierten Beschreibung durchgesetzt. Mit Recht wurde gefordert, dass Angaben zu den Sprecher-/ Schreibereinstellungen und den kommunikativen Funktionen von Phraseologismen in Wörterbüchern - unabhängig von ihrem Typ - unbedingt notwendig sind, um die hinsichtlich der lexikographischen Kodifizierung von Phraseologismen immer wieder beklagte Diskrepanz zwischen Sprachwirklichkeit und Wörterbuchpraxis zu mindern. ${ }^{13}$

Bei der Suche nach Äquivalenten sollte vom Begriff der funktionalen Äquivalenz ausgegangen werden. Dies impliziert, dass die ausgangsprachlichen Phraseologismen mit denen der Zielsprache in der gleichen kommunika- 
tiven Situation gebraucht werden können. Besteht der Äquivalentteil aus mehreren Ausdrücken, so gilt die Regelung, dass derjenige Ausdruck, der der deutschen Ausgangseinheit hinsichtlich der Bedeutung und Gebrauchsmöglichkeiten am ehesten entspricht, an erster Stelle erscheint. Wenn es zu einem deutschen Phraseologismus keinen adäquaten Ausdruck gibt, müssen im Äquivalentteil ergänzend Erläuterungen geboten werden. Diese erscheinen in Petit und stehen nach dem Äquivalent. Da ein Fremdsprachler nicht immer entscheiden kann, welche Konstituente bei der Lemmaansetzung in Frage kommt, wird im Wörterbuch mit Verweisangaben gearbeitet. Die Pfeile können nach der aktuellen Suchrichtung nach oben bzw. nach unten zeigen. Aufgrund vielfacher Fehler - wie auch aus Platzgründen - wäre von einer Mehrfachlemmatisierung abzuraten.

Im Folgenden wird die konzeptionelle Ausrichtung der Wörterbuchartikel für ein neues phraseologisches Wörterbuch Deutsch-Rumänisch vorgestellt. Die Einführung pragmatischer Parameter, die für das richtige Verständnis und den korrekten Gebrauch eines Phraseologismus ausschlaggebend sind, d.h. eine erweiterte Mikrostruktur, würde dem Benutzer Informationen bieten, die in den angenommenen Benutzungssituationen relevant sind. Zusatzangaben wie präzisierende und pragmatische Erläuterungen sichern eine differenziertere Beschreibung der Besonderheiten und der Gebrauchsbedingungen. Die verbesserte Kennzeichnung der Verwendungsweisen und eine differenziertere Markierungspraxis sind für die Qualität des mikrostrukturellen Profils des Nachschlagewerks ausschlaggebend.

Das vorgeschlagene Artikelprofil zur Verbesserung der rumänischen bilingualen Wörterbuchpraxis weist daher folgende lexikographische Parameter auf:

- Nennformangabe / rum. forma uzuală;

- Bedeutungserläuterung / rum. semnificaţie;

- Angaben zur sprachlichen Umgebung / rum. caracterizare [externe Valenz / rum. valenţa externă; z.B. jmd.; freie Angabe / rum. element facultativ; Negationsform(en) / forma de negare; z.B. nicht];

- Variationsangaben / rum. forme de varientare;

- pragmatische Angaben / rum. profil pragmatic [Charakterisierung der Gebrauchssituation; \pm mündlich / schriftlich / rum. indicaţii contextualpragmatice privind specificul situaţiei de comunicare; \pm oral / scris; Einstellung- / Bewertungsindikatoren / rum. evaluare; Emotionalitätsgrad / rum. gradul de emoţionalitate; Stilmarker / rum. nivelul stilistic; Charakterisierung der Sprecher-Hörer-Konstellation; z.B. \pm Vertrautheit, \pm formell, \pm hierarchisch, \pm symmetrisch / rum. relaţia vorbitor-receptor];

- kommunikativer Wert / rum. valoarea comunicativă;

- phraseosemantische Einordnung / rum. câmp frazeosemantic;

- Äquivalenzangabe(n) / rum. echivalent(e): fig. (rum. figurat; dt. bildhaft; übertragen); Stilmarker / rum. nivelul stilistic;

- Verweis / rum. trimitere la. 
Dieser Modellvorschlag ermöglicht durch die eingebrachten Angaben Ungenauigkeiten oder Fehlinterpretationen beim Benutzer weitgehend auszuschließen. Wie dieses Beschreibungsmodell, das eine verbesserte lexikographische Darstellung des aufgenommenen Sprachmaterials in einem neuen phraseologischen Wörterbuch Deutsch-Rumänisch anvisiert, in die lexikographische Praxis umgesetzt wird, soll im Folgenden an einem Probeartikel aufgezeigt werden. Dabei wird exemplarisch auf die Bearbeitung des Phraseologismus vom/aus dem Regen in die Traufe kommen eingegangen, um auch zu zeigen, wie einige Mängel der herkömmlichen rumänischen bilingualen Wörterbuchpraxis mit Deutsch (vgl. 2.1) behoben werden können. Die Eintragungen zu diesem Phraseologismus in den gängigen Wörterbüchern zu diesem Sprachenpaar sind äußerst knapp: vom/aus dem Regen in die Traufe kommen - a da din lac în puț (Roman 1993: 203) und a cădea/a da/a sări din lac în puţ — aus dem/vom Regen in die Traufe kommen (Mantsch/Anuţei/Kelp 1979: 289). Das verbesserte Artikelprofil für ein neues phraseologisches Wörterbuch bietet dem rumänischen Wörterbuchbenutzer unter dem Lemma Regen folgende Informationen:

jmd. kommt vom/aus dem Regen in die Traufe: a da de un rău mai mare în încercarea de a scăpa de un alt rău; a ajunge dintr-o situaţie critică în alta şi mai critică; \pm comunicare orală / + nemulţumire / + emoţionalitate; fam.; + familiaritate / \pm formal / ierarhic; DEZILUZIE / RESEMNARE / SUPĂRARE; câmp frazeosemantic: NEPLĂCERE / DECEPŢIE / DEZAMĂGIRE; [fig]; fam. a cădea / a da / a sări din lac în puţ; din rău în mai rău; a căuta pe dracul şi a găsi pe tată-său; trimitere la: Traufe $\uparrow$

Dieser Modellvorschlag bietet nicht nur zielsprachliche rumänische Äquivalente an, sondern erfasst auch Besonderheiten der kodifizierten deutschen phraseologischen Einheiten. Die Benutzerfreundlichkeit wird nicht nur durch den Einbezug pragmatischer Parameter bei der Gebrauchsmusterbeschreibung gewährleistet, sondern auch durch den Zugriff auf eine flexiblere, differenziertere Markierungsweise und die Zuordnung ausgangssprachlicher Fügungen zu einem phraseosemantischen Feld, womit die Suche des Benutzers nach dem geeigneten funktionalen Äquivalent vereinfacht wird. ${ }^{14}$

Die Anzahl der Angaben, die in einem Wörterbuch vorkommen, hängt von verschiedenen Faktoren (Aktiv-Passiv-Prinzip, Wörterbuchumfang, Benutzerbedürfnisse) ab. Dabei ist zu beachten, dass je stärker die aktive Funktion berücksichtigt wird, desto vielfältigere Informationen zum Kontext und zum Gebrauch geboten werden müssen. Über Äquivalenzangaben hinaus sollten in einem bilingualen aktiven Wörterbuch pragmatische Zusatzinformationen wie auch Informationen zur syntaktischen Einbettung geliefert werden.

Dieser Alternativvorschlag stellt bestimmte Anforderungen an seine Benutzer; er ist daher eher für einen Benutzer konzipiert worden, der sich der 
Mühe unterzieht, einen kompletten Wörterbuchausschnitt durchzuarbeiten und dabei auch die Hinweise zur Benutzung in der Einleitung nachzulesen. Die empirische Benutzungsforschung hat gezeigt, dass die Wörterbuchbenutzer die in den Nachschlagewerken dargebotenen Informationen nicht immer verstehen oder angemessen verwerten können. ${ }^{15}$ Gleichfalls muss darauf hingewiesen werden, dass nicht kühne Entwürfe, sondern zuverlässige Informationen für die Konzeption neuer Lexika bzw. bei der Planung von Neuauflagen ausschlaggebend sind. Man kann auch Wiegand (1998a: 193) nur zustimmen, wenn er feststellt:

Es ist sicherlich kein Verdienst, lexikographische Methoden und die aus deren Anwendung resultierende Praxis zu kritisieren und sie z.B. als unangemessen zu beurteilen [...]; es reich m.E. auch nicht aus, lediglich mehr oder weniger allgemeine Vorschläge $\mathrm{zu}$ machen oder weitgehende Forderungen $\mathrm{zu}$ formulieren, ohne Rücksicht darauf, ob sie in der Wörterbucharbeit praktikabel sind. Wer Lexikographen und deren Produkte kritisiert, sollte - wenigstens exemplarisch — eine alternative Praxis vormachen [Hervorhebung im Original], und zwar jene, die er selbst für die angemessenere hält.

Die Erstellung von aktiven phraseologischen Wörterbüchern, die mehr Informationen über eine beschränkte Auswahl von Phraseologismen bieten, ist eine zentrale Aufgabe der modernen Phraseographie. ${ }^{16}$ Für Neuauflagen vorhandener Wörterbücher oder für die Konzeption neuer Werke muss aus der Perspektive des Nichtmuttersprachlers und seiner Benutzerbedürfnisse für die rumänische phraseographische Praxis mit Deutsch eine verbesserte, benutzerfreundliche linguistische und metalexikographische Behandlung der Phraseologismen gesichert werden. Eine verbesserte Adressatenorientiertheit kann durch einen mikrostrukturellen Ausbau und die Ausarbeitung einer speziell auf die Bedürfnisse der Benutzer zugeschnittenen Mikrostruktur gesichert werden. Nicht nur durch eine verbesserte Konzeption der Artikelkörper, die dem modernen Stand der phraseologischen und phraseographischen Forschung entsprechen, sondern auch durch den Ausbau und die Umgestaltung der Umtexte wird die Benutzerfreundlichkeit zusätzlich erhöht. Dabei ist das Heranziehen zuverlässiger Vorgängerwerke, Fachquellen und Textkorpora grundlegend wie auch die Ausarbeitung einer für phraseologische Inventareinheiten adäquaten Beschreibungsweise.

Mit der Behebung der hier aufgezeigten Versäumnisse hinsichtlich der lexikographischen Konzeption und Ausgestaltung der Wörterbücher würde nicht nur ein Beitrag zur aktiven Phraseographie geleistet werden. Die Ausarbeitung eines wissenschaftlich und empirisch abgesicherten lexikographischen Modellvorschlags, der sich konzeptionell auf die Ergebnisse der theoretischen und praktischen Phraseologie/Phraseographie stützt, um verlässliche Auskunft bei der Produktion/Rezeption von Texten zu bieten, würde auch die Erforschung der Phraseologie dieser Sprachen im internationalen Vergleich erleichtern. 


\section{Schlussbemerkungen}

Vorliegender Beitrag hat Fragen der Erarbeitung von zweisprachigen Wörterbüchern sowie lexikographische Standards diskutiert und deren Berücksichtigung/Verwirklichung in der rumänischen praktischen Lexikographie ausdrücklich gefordert, um dem Benutzer ein brauchbares Hilfsmittel an die Hand zu geben oder dem Übersetzer zuverlässigere Äquivalenzzuordnungen zu ermöglichen. Um der zweisprachigen lexikographischen Praxis wertvolle Anregung zu vermitteln, wurde die Einbindung der aus (sprach)praktischer und phraseologischer Sicht relevanten Angaben zu den Gebrauchsbeschränkungen und -bedingungen an einem Modellvorschlag aufgezeigt.

Der auf die herkömmliche rumänische bilinguale lexikographische Praxis mit Deutsch ausgerichtete Beitrag hat gezeigt, welche Domänen phraseographischer Relevanz anzusetzen sind, um wissenschaftlich und empirisch abgesicherte phraseologische Nachschlagewerke ausarbeiten zu können. Als Ergebnis der kritischen Durchsicht gängiger Wörterbücher zu diesem Sprachenpaar und bei der Prüfung ihrer konzeptionellen Ausrichtung lassen sich Defizite festhalten, die für die Orientierung in rezeptiven wie in produktiven Nachschlagesituationen ausschlaggebend sind. Insgesamt kann eine benutzerunfreundliche und unangemessene Präsentierung des phraseologischen Materials - Inkonsequenzen bei der Festlegung der Nennform, der Handhabung lexikalischer Varianten oder bei Äquivalenzangaben - ausgemacht werden wie auch zahlreiche Verstöße gegen die lexikographische Akribie.

Angesichts lexikographischer Leistungen der bilingualen Speziallexikographie weltweit und der Brauchbarkeit vorhandener Wörterbücher zum Sprachenpaar Deutsch und Rumänisch aus heutiger Sicht ist ersichtlich, dass die phraseographische Erfassung der Sprachen Deutsch und Rumänisch ein bis dato stark vernachlässigtes Feld der praktischen Phraseographie ist, das es zu fördern gilt.

Da die gängigen rumänischen bilingualen Wörterbücher mit Deutsch einer Aktualisierung bedürfen und auf dem Wörterbuchmarkt längst vergriffen sind, ist die Entwicklung moderner bilingualer phraseologischer Nachschlagewerke mit Deutsch als Ausgangs- oder Zielsprache eine noch nachzuholende Aufgabe der rumänischen Phraseographie. Mit dem hier vorgestellten Alternativvorschlag war das Bestreben verbunden, die lexikographische Praxis anzuregen und $\mathrm{zu}$ verbessern, wobei auch einer adäquaten lexikographischen Darstellung phraseologischer Einheiten Rechnung getragen wurde. Eine verbesserte Erfassung der Gebrauchsweisen der Phraseologismen würde auch den Rückstand der rumänischen bilingualen Wörterbuchpraxis mit Deutsch aufholen und implizite auch eine erhöhte Qualität des Wörterbuchs und dessen Benutzerfreundlichkeit sichern.

Unter Bezugnahme auf den aktuellen Stand der rumänischen lexikographischen Praxis mit Deutsch ist ersichtlich, dass die Erstellung eines modernen Nachschlagewerks mit Deutsch als Ausgangs- oder Zielsprache eine dringende Aufgabe der rumänischen Phraseographie darstellt. 


\section{Anmerkungen}

1. $\quad \mathrm{Zu}$ allgemeinen Fragen der lexikographischen Behandlung von Phraseologismen in Wörterbüchern vgl. u.a. Barz/Henning/Korhonen (2005), Burger et al. (2007), Blanco (2009) oder Blanco et al. (2010).

2. Vgl. dazu z.B. Bergenholtz/Tarp (2003).

3. Vgl. hierzu die Ausführungen bei Schemann (z.B. 1989, 1991), Martin (2001) oder Ptashnyk (2003).

4. Sava (2010). Vgl. Mantsch/Anuţei/Kelp (Rumänisch-Deutsch; 1979) und Roman (DeutschRumänisch; 1993).

5. Obwohl der Wörterbuchbenutzung in der lexikographischen Diskussion ein breiter Raum eingeräumt wurde, sind Umfang und Ausgestaltung der Umtexte in der deutschsprachigen Wörterbuchlandschaft sporadisch Gegenstand metalexikographischer Reflexionen gewesen. Die kritischen Anmerkungen zur phraseographischen Wörterbuchpraxis, die in der Forschung anzutreffen sind, betreffen vornehmlich Probleme der Lemmatisierung, Nennformgestaltung und Gebrauchsmarkierung phraseologischer Einheiten.

6. Hierfür sind u.a. die Textkorpora des Instituts für deutsche Sprache in Mannheim zu empfehlen.

7. Fragen der lexikographischen Bearbeitung von Phraseologismen aus bilingualer Perspektive sind Diskussionsgegenstand u.a. bei Filipenko (2002, Deutsch-Russisch), Martin (2001, DeutschSpanisch), Petelenz (2001, Deutsch-Polnisch), Cheon (1998, Deutsch-Koreanisch). Manche Autoren erläutern lexikographische Standards der bilingualen Phraseographie am Material phraseologischer Untergruppen (Farø 2002; Deutsch-Dänisch/Somatismen) oder rücken linguistisch-lexikographisch relevante Kodifizierungsbereiche in den Mittelpunkt der Betrachtungen (z.B. pragmatische Angaben in einem zweisprachigen Lernerwörterbuch DeutschBulgarisch, Drumeva, 2001). Mit der Spezifik der bilingualen Wörterbuchpraxis befassen sich auch die Beiträge von Petkov (2001) oder Korhonen (z.B. 2004), während eine eingehende Besprechung zur Theorie und Praxis der Phraseographie Schemann (1991) leistet. Der Sammelband von Blanco et al. (2010) dokumentiert den aktuellen Forschungsstand und gibt wichtige Impulse für die Weiterentwicklung der ein- und zweisprachigen Phraseographie des 21. Jahrhunderts.

8. Die neuere Phraseologieforschung fordert die Einbeziehung peripherer phraseologischer Bereiche (z.B. Funktionsverbgefüge, Routineformeln) und befürwortet eine weite Phraseologieauffassung. Für die Lemmaauswahl sollten Textkorpora, allgemeine und spezielle Wörterbücher herangezogen werden aber auch verschiedene Quellen nichtlexikographischer Art wie Lehrbücher.

9. Vgl. dazu Földes (z.B.1996) oder Ptashnyk (2003).

10. Dazu auch Sava (2015).

11. Vgl. hierzu auch Martin (2001: 142).

12. Näheres dazu bei Hausmann (1989: 649).

13. Vgl. hierzu u.a. Blanco et al. (2010).

14. Dazu u.a. Dobrovols'kij (1997).

15. Zur Benutzung von Wörterbüchern vgl. Wiegand (1998) oder Engelberg/Lemnitzer (2009).

16. Als innovativer Ansatz für die Erforschung fester Wortverbindungen gilt die Methode der statistischen Kookkurrenzanalyse, die in den 1980er-Jahren am Institut für deutsche Sprache 
in Mannheim entwickelt wurde. Steyer präsentiert in mehreren Arbeiten (z.B. 2000 und 2004) das linguistische Modell, die Korpusmethodik und die lexikographischen Perspektiven dieses Ansatzes.

\section{Das Acknowledgement}

Dieser Artikel entstand im Rahmen des von der Lucian-Blaga-Universität Sibiu / Hermannstadt geförderten Projekts LBUS-IRG-2017-03 (Laufzeit: 2017-2019).

\section{Literatur}

Barz, Irmhild, Henning Bergenholtz und Jarmo Korhonen (Hrsg.). 2005. Schreiben, Verstehen, Übersetzen, Lernen. Zu ein- und zweisprachigen Wörterbüchern mit Deutsch. Finnische Beiträge zur Germanistik 14. Frankfurt [etc.]: Peter Lang.

Blanco, Carmen Mellado (Hrsg.). 2009. Theorie und Praxis der idiomatischen Wörterbücher. Tübingen: Max Niemeyer.

Blanco, Carmen Mellado, Patricia Buján, Claudia Herrero, Nely Iglesias und Ana Mansilla (Hrsg.). 2010. La fraseografía del S. XXI. Nuevas propuestas para el español y el alemán. Romanistik 6. Berlin: Frank \& Timme.

Bergenholtz, Henning und Sven Tarp. 2003. Two Opposing Theories: On H.E. Wiegand's Recent Discovery of Lexicographic Functions. Hermes. Journal of Linguistics 31: 171-196.

Burger, Harald. 1992. Phraseologie im Wörterbuch. Überlegungen aus germanistischer Perspektive. Eismann, Wolfgang und Jürgen Petermann (Hrsg.). 1992. Studia phraseologica et alia. Festschrift für J. Matešić zum 65. Geburtstag: 33-51. Specimina Philologiae Slavicae. Supplementband 31. München: Sagner.

Burger, Harald, Dmitrij Dobrovol'skij, Peter Kühn und Neal R. Norrick (Hrsg.). 2007. Phraseologie. Ein internationales Handbuch der zeitgenössischen Forschung [...]. Berlin/New York: Walter de Gruyter.

Cheon, Mi-Ae. 1998. Zur Konzeption eines phraseologischen Wörterbuchs für den Fremdsprachler: Am Beispiel Deutsch-Koreanisch. Lexicographica. Series Maior 89. Tübingen: Max Niemeyer.

Dobrovol'skij, Dmitrij. 1997. Prototypentheoretische Ansätze bei der Beschreibung der Idiomatik. Barz, Irmhild und Marianne Schröder (Hrsg.). 1997. Nominationsforschung im Deutschen. Festschrift für W. Fleischer zum 75. Geburtstag: 157-165. Frankfurt [etc.]: Peter Lang.

Drumeva, Stanislava. 2001. Pragmatische Angaben in einem zweisprachigen Lernerwörterbuch mit Deutsch und Bulgarisch. Igla, Birgit, Pavel Petkov und Herbert Ernst Wiegand (Hrsg.). 2001: 21-40.

Engelberg, Stefan und Lothar Lemnitzer. 2009. Lexikographie und Wörterbuchbenutzung. 4. Aufl. Tübingen: Stauffenburg.

Farø, Ken. 2002. Somatismen als Problem der dänischen und deutschen Lexikographie. Gottlieb, Henrik, Jens Erik Mogensen und Arne Zettersten (Hrsg.). 2002. Symposium on Lexicography X. Proceedings of the Tenth International Symposium on Lexicography May 4-6, 2000, at the University of Copenhagen: 107-124. Lexicographica. Series Maior 109. Tübingen: M. Niemeyer. 
Filatkina, Natalia. 2007. Pragmatische Beschreibungsansätze. Burger, Harald, Dmitrij Dobrovol'skij, Peter Kühn und Neal R. Norrick (Hrsg.). 2007: 132-158.

Filipenko, Tat'jana V. 2002. Beschreibung der Idiome in einem zweisprachigen Idiomatik-Wörterbuch (Deutsch-Russisch). Vollstedt, Marina (Hrsg.). 2002. Das Wort. Germanistisches Jahrbuch GUS: 43-62.

Földes, Csaba. 1996. Gesucht ... und gefunden? Idiomlexika und Deutsch als Fremdsprache. Fremdsprache Deutsch 15: 64-67.

Hausmann, Franz Josef. 1989. Die Markierung im allgemeinen einsprachigen Wörterbuch: eine Übersicht. Hausmann, Franz Josef, Oskar Reichmann, Herbert Ernst Wiegand und Ladislav Zgusta (Hrsg.). 1989: 649-657.

Hausmann, Franz Josef, Oskar Reichmann, Herbert Ernst Wiegand und Ladislav Zgusta (Hrsg.). 1989-1991. Wörterbücher. Dictionaries. Dictionnaires. Ein internationales Handbuch zur Lexikographie [...]. Handbücher zur Sprach- und Kommunikationswissenschaft 5.1-5.3. Berlin/New York: Walter de Gruyter.

Igla, Birgit, Pavel Petkov und Herbert Ernst Wiegand (Hrsg.). 2001. Theoretische und praktische Probleme der Lexikographie. 1. Internationales Kolloquium zur Wörterbuchforschung am Institut Germanicum der St. Kliment Ohridski-Universität Sofia, 7. bis 8. Juli 2000. Germanistische Linguistik 161-162/2001. Hildesheim/Zürich/New York: Georg Olms.

Korhonen, Jarmo. 2004. Duden 11 - Nutzungserfahrungen aus der DaF-Perspektive. Steyer, Kathrin (Hrsg.). 2004. Wortverbindungen - mehr oder weniger fest. Jahrbuch des Instituts für Deutsche Sprache 2003: 360-392. Berlin: Walter de Gruyter.

Mantsch, Heinrich, Mihai Anuţei und Helmut Kelp. 1979. Dicţionar frazeologic român-german. Bucureşti: Ştiinţifică şi Enciclopedică.

Martin, Luis. 2001. Phraseologie im zweisprachigen Wörterbuch Deutsch-Spanisch. Hispano-Americana. Geschichte, Sprache, Literatur 28. Frankfurt [etc.]: Peter Lang.

Mudersbach, Klaus. 1998. Ein Vorschlag zur Beschreibung von Phrasemen auf der Basis eines universalen pragmatischen Modells. Wiegand, Herbert Ernst (Hrsg.). 1998. Wörterbücher in der Diskussion III. Vorträge aus dem Heidelberger Lexikographischen Kolloquium. Lexicographica. Series Maior 84: 319-347. Tübingen: Max Niemeyer.

Petelenz, Krzysztof. 2001. Standardisierung der Lexikoneinträge für ein neues großes deutschpolnisches und polnisch-deutsches Wörterbuch. Wiegand, Herbert Ernst (Hrsg.). 2001. Studien zur zweisprachigen Lexikographie mit Deutsch VII. Germanistische Linguistik 159-160/2001. Hildesheim/Zürich/New York: Georg Olms.

Petkov, Pavel. 2001. Zum Problem der Äquivalenzbeziehung und der lexikographischen Lücke in zweisprachigen Wörterbüchern. Igla, Birgit, Pavel Petkov und Herbert Ernst Wiegand (Hrsg.). 2001: 73-81.

Ptashnyk, Stefaniya. 2003. Wie hart ist die phraseographische Nuss? Überlegungen zu phraseologischen Wörterbüchern aus der Sicht der ukrainischen Germanistik. Wierlacher, Alois, Konrad Ehlich, Ludwig M. Eichinger et al. (Hrsg.). 2003. Jahrbuch Deutsch als Fremdsprache. Intercultural German Studies. Bd. 29: 31-54. München: Iudicium.

Roman, Alexandru. 1993. Dicţionar frazeologic german-român. Bucureşti: Teora.

Sava, Doris. 2010. Defizite der rumänischen bilingualen Phraseographie mit Deutsch. Germanistische Beiträge 26: 185-222. 
Sava, Doris. 2015. Linguistische Zugänge zur phraseographischen Beschreibungspraxis. Einsatzmöglichkeiten für die rumänische bilinguale Phraseographie mit Deutsch. Forschungen zur Volks- und Landeskunde 58: 159-176.

Schemann, Hans. 1989. Das phraseologische Wörterbuch. Hausmann, Franz Josef, Oskar Reichmann, Herbert Ernst Wiegand und Ladislav Zgusta (Hrsg.). 1989: 1019-1032.

Schemann, Hans. 1991. Die Phraseologie im zweisprachigen Wörterbuch. Hausmann, Franz Josef, Oskar Reichmann, Herbert Ernst Wiegand und Ladislav Zgusta (Hrsg.). 1991: 2789-2794.

Steyer, Kathrin. 2000. Usuelle Wortverbindungen des Deutschen. Linguistisches Konzept und lexikographische Möglichkeiten. Deutsche Sprache 28: 101-125.

Steyer, Kathrin. 2004. Kookkurrenz. Korpusmethodik, linguistisches Modell, lexikographische Perspektiven. Steyer, Kathrin (Hrsg.). 2004. Wortverbindungen - mehr oder weniger fest. Jahrbuch des Instituts für Deutsche Sprache 2003: 87-116. Berlin: Walter de Gruyter.

Wiegand, Herbert Ernst. 1998. Wörterbuchforschung. Untersuchungen zur Wörterbuchbenutzung, zur Theorie, Geschichte, Kritik und Automatisierung der Lexikographie. 1. Teilband. Berlin/New York: Walter de Gruyter.

Wiegand, Herbert Ernst. 1998a. Pragmatische Informationen in neuhochdeutschen Wörterbüchern. Ein Beitrag zur praktischen Lexikologie. Wiegand, Herbert Ernst (Hrsg.). 1998. Studien zur neuhochdeutschen Lexikographie I: 139-271. Hildesheim/Zürich/New York: Georg Olms. (= Germanistische Linguistik 3-4/79). 